\title{
c-ALGEBRABILITY OF PATHOLOGICAL SETS OF PRODUCT INTEGRABLE FUNCTIONS
}

\author{
FATEMEH FARMANESH, ALI FAROKHINIA* \\ Department of Mathematics, Shiraz Branch, Islamic Azad University, Shiraz, Iran \\ *Corresponding author: alifarokinia@gmail.com
}

\begin{abstract}
In this paper we investigate linear algebraic structures in the set of product integrable matrixvalued functions and find $\mathfrak{c}$-generated algebras in $L\left([a, b], \mathbb{R}^{n \times n}\right) \backslash L^{*}\left([a, b], \mathbb{R}^{n \times n}\right)$ and $D\left([a, b], \mathbb{R}^{n \times n}\right) \backslash L\left([a, b], \mathbb{R}^{n \times n}\right)$.
\end{abstract}

\section{INTRODUCTION}

If $X$ is a vector space, a subset $M$ of $X$ is called lineable if $M \cup\{0\}$ contains an infinite dimensional vector space. If $X$ is a linear algebra and $M \subseteq X$, one calls $M$ a $\kappa$-algebrable set if $M \cup\{0\}$ contains a $\kappa$-generated algebra, that is, an algebra which has a minimal system of generators of cardinality $\kappa$. These notions were coined by V.I. Guariy [1,9] and then became a criterion for measuring how much large linear algebraic structures could be found in a set of functions with weird properties (see [2,6-8]).

Another criterion is the concept of strong algebrability introduced by Glab and Bartoszewicz in [5]. Let $\kappa$ be a cardinal number and $X$ be a linear commutative algebra. A subset $M$ of $X$ is called strongly $\kappa$-algebrable if $M \cup\{0\}$ contains a $\kappa$-generated algebra isomorphic to a free algebra.

In this paper we seek a linear algebraic structures in the spaces of product integrable function. The notion of product integral has been introduced by Vito Volterra about the end of the 19th century, who studied

Received 2018-04-26; accepted 2018-08-02; published 2018-09-05.

2000 Mathematics Subject Classification. 47A16, 47L10.

Key words and phrases. algebrable; Lebesgue integrable; product integrable.

(C)2018 Authors retain the copyrights of their papers, and all open access articles are distributed under the terms of the Creative Commons Attribution License. 
linear systems of differential equations

$$
\begin{aligned}
& W^{\prime}(t)=A(t) W(t), t \in[a, b] \\
& W(a)=I
\end{aligned}
$$

where $I$ is the identity matrix, $A:[a, b] \rightarrow \mathbb{R}^{n \times n}$ is a given continuous function and $W:[a, b] \rightarrow \mathbb{R}^{n \times n}$ is the unknown function (see [17]). Later, Ludwig Schlesinger introduced the definition of the Riemann product integral as follows: Given a tagged partition of an interval $[a, b]$, which is a collection of point-interval pairs $D=\left(\xi_{i},\left[t_{i-1}, t_{i}\right]\right)_{i=1}^{m}$, where $a=t_{0} \leq t_{1} \leq \ldots \leq t_{m}=b$ and $\xi_{i} \in\left[t_{i-1}, t_{i}\right]$ for every $i \in\{1,2, \ldots, m\}$. We refer to $t_{0}, t_{1}, \ldots, t_{m}$ as the division points of $D$, while $\xi_{1}, \xi_{2}, \ldots, \xi_{m}$ are the tags of $D$.

Remark 1.1. If we replace $\xi_{i} \in\left[t_{i-1}, t_{i}\right]$ by $\xi_{i} \in[a, b]$, then the collection $D$ is called a free tagged partition. Given a function $\delta:[a, b] \rightarrow \mathbb{R}^{+}$(called a gauge on $[a, b]$ ), a free tagged partition is called $\delta$-fine if

$$
\left[t_{i-1}, t_{i}\right] \subset\left(\xi_{i}-\delta\left(\xi_{i}\right), \xi_{i}+\delta\left(\xi_{i}\right)\right), i=\{1,2, \ldots, m\}
$$

Now consider a matrix function $A:[a, b] \rightarrow \mathbb{R}^{n \times n}$ with entries $\left\{a_{i j}\right\}_{i, j=1}^{n}$. Put

$$
\Delta t_{i}=t_{i}-t_{i-1}, i=1,2, \ldots, m, v(D)=\max _{1 \leq i \leq m} \Delta t_{i}
$$

and define

$$
\begin{aligned}
P(A, D) & =\prod_{i=1}^{m}\left(I+A\left(\xi_{i}\right) \Delta t_{i}\right) \\
& =\left(I+A\left(\xi_{1}\right) \Delta t_{1}\right)\left(I+A\left(\xi_{2}\right) \Delta t_{2}\right) \ldots\left(I+A\left(\xi_{m}\right) \Delta t_{m}\right) .
\end{aligned}
$$

In case the $\operatorname{limit}_{v(D) \rightarrow 0} P(A, D)$ exists, it is called the Riemann product integral of the function $A$ on the interval $[a, b]$ and is denoted by the symbol $(I+A(t) d t) \prod_{a}^{b}$.

In this paper $R\left([a, b], \mathbb{R}^{n \times n}\right)$ denotes the set of all Riemann product integrable functions.

Utilizing step functions Schlesinger generalized this definition and introduced the Lebesgue product integral (see $[11,12,16])$. Let us recall some facts that will be needed:

1. A function $A:[a, b] \rightarrow \mathbb{R}^{n \times n}$ is called a step function if there exists numbers $a=t_{0}<t_{1}<\ldots<$ $t_{m}=b$ such that $A$ is constant function on every interval $\left(t_{k-1}, t_{k}\right), k=1,2, \ldots, m$.

2. For $A \in \mathbb{R}^{n \times n}$ we will use the operator norm $\|A\|=\sup \{\|A x\|:\|x\| \leq 1\}$, where $\|A x\|$ and $\|x\|$ denote the Euclidean norms of vectors $A x, x \in \mathbb{R}^{n}$.

3. A sequence of functions $\left\{A_{k}:[a, b] \rightarrow \mathbb{R}^{n \times n}\right\}_{k \in \mathbb{N}}$ is called uniformly bounded if there exists a number $M \in \mathbb{R}$ such that $\left\|A_{k}(x)\right\| \leq M$ for all $k \in \mathbb{N}$ and all $x \in[a, b]$. 
Theorem 1.1. [16, Lemma 3.5.4 and Theorem 3.5.5] Let $A_{k}:[a, b] \rightarrow \mathbb{R}^{n \times n}, k \in \mathbb{N}$, be a uniformly bounded sequence of step functions such that $\lim _{k \rightarrow \infty} A_{k}(x)=A(x)$ a.e. on $[a, b]$. Then

$$
\lim _{k \rightarrow \infty}\left\|A_{k}-A\right\|_{1}=\lim _{k \rightarrow \infty} \int_{a}^{b}\left\|A_{k}(x)-A(x)\right\| d x=0,
$$

and the limit $\lim _{k \rightarrow \infty}\left(I+A_{k}(x) d x\right) \prod_{a}^{b}$ exists and is independent of the choice of the sequence $\left\{A_{k}\right\}$.

Definition 1.2. [16, Definiton 3.5.6] Consider the function $A:[a, b] \rightarrow \mathbb{R}^{n \times n}$. Assume there exists a uniformly bounded sequence of step functions $A_{k}:[a, b] \rightarrow \mathbb{R}^{n \times n}$ such that $\lim _{k \rightarrow \infty} A_{k}(x)=A(x)$ a.e. on $[a, b]$, then the function $A$ is called Lebesgue product integrable and we define

$$
(I+A(x) d x) \prod_{a}^{b}=\lim _{k \rightarrow \infty}\left(I+A_{k}(x) d x\right) \prod_{a}^{b} .
$$

The symbole $L^{*}\left([a, b], \mathbb{R}^{n \times n}\right)$ denotes the set of all Lebesgue product integrable functions. It is easy to show that

$$
L^{*}\left([a, b], \mathbb{R}^{n \times n}\right)=\left\{A:[a, b] \rightarrow \mathbb{R}^{n \times n}: A \text { is measurable and bounded }\right\} .
$$

Let us recall that a function $A:[a, b] \rightarrow \mathbb{R}^{n \times n}$ is called Bochner intagrable if there is a sequence of simple functions $A_{k}:[a, b] \rightarrow \mathbb{R}^{n \times n}, k \in \mathbb{N}$ such that $\lim _{k \rightarrow \infty} A_{k}(t)=A(t)$ a.e. on $[a, b]$ and

$$
\lim _{k \rightarrow \infty}\left\|A_{k}-A\right\|_{1}=0
$$

Thus by Theorem 1.1 and Definition 1.2, each $A \in L^{*}\left([a, b], \mathbb{R}^{n \times n}\right)$ is Bochner intagrable.

After that Schlesinger extended the definition of $L^{*}\left([a, b], \mathbb{R}^{n \times n}\right)$ to all matrix functions with Lebesgue integrable (not necessarily bounded) entries and used the next symbole:

$$
L\left([a, b], \mathbb{R}^{n \times n}\right)=\left\{A:[a, b] \rightarrow \mathbb{R}^{n \times n}:(L) \int_{a}^{b}\|A(x)\| d x<\infty\right\} .
$$

The symbole (L) estands for the Lebesgue integral. Taking account of Theorem 1.1 it is natural to state the following definition.

Definition 1.3. [16, Definiton 3.8.1] A function $A:[a, b] \rightarrow \mathbb{R}^{n \times n}$ is called product integrable if there exists a sequence of step functions $\left\{A_{k}\right\}$ such that

$$
\lim _{k \rightarrow \infty}\left\|A_{k}-A\right\|_{1}=0 .
$$

We define

$$
(I+A(x) d x) \prod_{a}^{b}=\lim _{k \rightarrow \infty}\left(I+A_{k}(x) d x\right) \prod_{a}^{b}
$$


Remark 1.2. Since step functions belong to the complete space $L\left([a, b], \mathbb{R}^{n \times n}\right)$, every product integrable function also belongs to $L\left([a, b], \mathbb{R}^{n \times n}\right)$. Moreover, step functions form a dense subset in this space, and hence $(I+A(x) d x) \prod_{a}^{b}$ exists if and only if $A \in L\left([a, b], \mathbb{R}^{n \times n}\right)$, i.e., the Lebesgue integral $\int_{a}^{b}\|A(t)\| d t$ is finite.

Concerning the above definitions of product integral we have the following chain of strict inclusions:

$$
R\left([a, b], \mathbb{R}^{n \times n}\right) \subset L^{*}\left([a, b], \mathbb{R}^{n \times n}\right) \subset L\left([a, b], \mathbb{R}^{n \times n}\right) .
$$

\section{The exponential Function and the product integral}

Recall that for every $A \in \mathbb{R}^{n \times n}$ the matrix exponential is defined by $e^{A}=\sum_{k=0}^{\infty} \frac{A^{k}}{k !}$.

Theorem 2.1. [16, Theorem 3.2.2] Consider a Riemann integrable function $A:[a, b] \rightarrow \mathbb{R}^{n \times n}$. Then

$$
\lim _{v(D) \rightarrow 0} \prod_{k=1}^{m} e^{A\left(\xi_{k}\right) \Delta t_{k}}=\lim _{v(D) \rightarrow 0} \prod_{k=1}^{m}\left(I+A\left(\xi_{k}\right) \Delta t_{k}\right)=(I+A(t) d t) \prod_{a}^{b},
$$

where partitions are as in introduction.

Remark 2.1. If $A \in L^{*}\left([a, b], \mathbb{R}^{n \times n}\right)$ and $\left\{A_{k}\right\}_{k=1}^{\infty}$ is a uniformly bounded sequence of step functions in $L^{*}\left([a, b], \mathbb{R}^{n \times n}\right)$ such that $A_{k} \rightarrow$ A a.e. on $[a, b]$, then by $\left[16\right.$, Theorem 3.6.3] we have $(I+A(x) d x) \prod_{a}^{b}=\lim _{k \rightarrow \infty}(I+$ $\left.A_{k}(x) d x\right) \prod_{a}^{b}$. Now every function $A_{k}$ is associated with a partition

$$
D_{k}: a=t_{0}^{k}<t_{1}^{k}<\ldots<t_{m(k)}^{k}=b
$$

such that

$$
A_{k}(x)=A_{j}^{k}, x \in\left(t_{j-1}^{k}, t_{j}^{k}\right)
$$

and

$$
\lim _{k \rightarrow \infty} v\left(D_{k}\right)=0
$$

So by the definition of Lebesgue product integrable functions,

$$
(I+A(x) d x) \prod_{a}^{b}=\lim _{k \rightarrow \infty}\left(I+A_{k}(x) d x\right) \prod_{a}^{b}=\lim _{k \rightarrow \infty} \prod_{j=1}^{m(k)} \exp \left(A_{j}^{k} \Delta t_{j}^{k}\right) .
$$

Moreover Schlesinger in [16, p. 485-486] proved the product integral might be also calculated as

$$
(I+A(x) d x) \prod_{a}^{b}=\lim _{k \rightarrow \infty} \prod_{j=1}^{m(k)}\left(I+\left(A_{j}^{k} \Delta t_{j}^{k}\right) .\right.
$$

We remark that each $A \in L^{*}\left([a, b], \mathbb{R}^{n \times n}\right)$ is Bochner integrable and hence the product integrals $\prod_{a}^{b} \exp (A(t) d t$ and $\prod_{a}^{b}(I+A(t) d t)$ exist and equal to each other; see [13, Theorem 14, Theorem 16]. Thus according to the previous discussion, Theorem 2.1 holds for all $A \in L^{*}\left([a, b], \mathbb{R}^{n \times n}\right)$. 
Now cosider a function $A \in L\left([a, b], \mathbb{R}^{n \times n}\right)$. By the definition 1.3 there exists a sequence of step functions $\left\{A_{k}\right\}_{k=1}^{\infty}$ such that

$$
\lim _{k \rightarrow \infty}\left\|A_{k}-A\right\|_{1}=0 \text { and }(I+A(t) d t) \prod_{a}^{b}=\lim _{k \rightarrow \infty}\left(I+A_{k}(t) d t\right) \prod_{a}^{b} .
$$

Thus Theorem 2.1 does also hold for $A \in L\left([a, b], \mathbb{R}^{n \times n}\right)$. So we can state the next theorem.

Theorem 2.2. Let $A:[a, b] \rightarrow \mathbb{R}^{n \times n}$ be a matrix function and $A \in L\left([a, b], \mathbb{R}^{n \times n}\right)$, then $\exp \circ A$ is product integrable.

\section{LeBESGue PRoduct INTEGRABLE FUnCtions}

The next definition and theorem provide important tools for proving the existence of infinitely generated algebras in the family of real or complex functions.

Definition 3.1 ( [3]). We say that a function $f: \mathbb{R} \rightarrow \mathbb{R}$ is an exponential-like function (of rank $m$ ) whenever $f$ is given by $f(x)=\sum_{i=1}^{m} a_{i} e^{b_{i} x}$ for some distinct nonzero real numbers $b_{1}, b_{2}, \ldots, b_{m}$ and some nonzero real numbers $a_{1}, a_{2}, \ldots, a_{m}$.

Theorem 3.2 $([3,4])$. Let $\mathcal{F} \subset \mathbb{R}^{[0,1]}$ and assume that there exists a function $F \in \mathcal{F}$ such that foF $\in \mathcal{F} \backslash\{0\}$ for every exponential-like function $f: \mathbb{R} \rightarrow \mathbb{R}$. Then $\mathcal{F}$ is strongly c-algebrable. More exactly, if $H \subset \mathbb{R}$ is a set of cardinality $\mathfrak{c}$ and linearly independent over the rationals $\mathbb{Q}$, then $\exp \circ(r F), r \in H$, are free generators of an algebra contained in $\mathcal{F} \cup\{0\}$.

Note that in all proofs we apply Theorem 3.2

Theorem 3.3. The set of Riemann real valued integrable functions is strongly c-algebrable.

Proof. Volterra in [17] showed that the Riemann integrable functions are product integrable, thus by Theorem 2.1 and Theorem 3.2 the proof follows.

Theorem 3.4. The set of real valued Lebesgue integrable functions is strongly c-algebrable.

Proof. Schlesinger in $[12,16]$ showed the product integrability of Lebesgue integrable functions. So by Theorem 2.2 and Theorem 3.2, the proof is complete.

Theorem 3.5. The set $L\left([a, b], \mathbb{R}^{n \times n}\right) \backslash L^{*}\left([a, b], \mathbb{R}^{n \times n}\right)$ is strongly c-algebrable.

Proof. Let $A:[0,1] \rightarrow \mathbb{R}^{n \times n}$ be given by $A(x)=\left(a_{i j}(x)\right)_{i, j=1}^{n}$ such that for each $i, j=1,2, . ., n$,

$$
a_{i j}(x)=\left\{\begin{array}{cc}
\frac{1}{\sqrt{x}} & x \in(0,1] \\
0 & x=0
\end{array}\right.
$$


So for some $y \in \mathbb{R}^{n \times 1}$ and $\|y\| \leq 1$,

$$
\begin{gathered}
A(x) y=\left(\begin{array}{ccc}
a_{11} & \ldots & a_{1 n} \\
\vdots & \ddots & \vdots \\
a_{n 1} & \cdots & a_{n n}
\end{array}\right)\left(\begin{array}{c}
y_{1} \\
\vdots \\
y_{n}
\end{array}\right)=\left(\begin{array}{c}
a_{11} y_{1}+a_{12} y_{2}+\cdots+a_{1 n} y_{n} \\
\vdots \\
a_{n 1} y_{1}+a_{n 2} y_{2}+\cdots+a_{n n} y_{n}
\end{array}\right), \\
\|A(x) y\| \geq \sqrt{\frac{n}{x}\left(y_{1}+\cdots+y_{n}\right)^{2}} \geq \frac{1}{x}, x \in(0,1] .
\end{gathered}
$$

Thus $A$ is not bounded and so $A$ and $\exp \circ(A)$ are not in $L^{*}\left([0,1], \mathbb{R}^{n \times n}\right)$. Now let $A_{m}:[0,1] \rightarrow \mathbb{R}^{n \times n}$ be given by $\left.A_{m}(x)=\stackrel{(m)}{\left(b_{i j}\right.}(x)\right)_{i, j=1}^{n}$ such that for each $i, j=1,2, . ., n$,

$$
\stackrel{(m)}{b_{i j}}(x)=\left\{\begin{array}{cc}
0 & x \in\left[0, \frac{1}{m}\right] \\
\frac{1}{\sqrt{x}} & x \in\left(\frac{1}{m}, 1\right]
\end{array}\right.
$$

Given an arbitrary $i$ and $j$, and note that for $m \geq 2, \stackrel{(m)}{b} b_{i j}(x)$ is Lebesgue integrable on $[0,1]$. Since $\lim _{m \rightarrow \infty} \stackrel{(m)}{b}_{i j}(x)=a_{i j}(x)$ for each $x \in[0,1]$, so by the Monotone Convergence Theorem $a_{i j}(x)$ is Lebesgue integrable. Thus $A$ and $\exp \circ(A)$ are in $L\left([0,1], \mathbb{R}^{n \times n}\right)$ so $f \circ(A)$ is in $L\left([0,1], \mathbb{R}^{n \times n}\right)$, for every exponentiallike function $f$, and the proof is complete by Theorem 3.2 .

Theorem 3.6. The set of $L\left([a, b], \mathbb{R}^{n \times n}\right) \backslash R\left([a, b], \mathbb{R}^{n \times n}\right)$ is $\mathfrak{c}$-algebrable.

Proof. Since $L\left([a, b], \mathbb{R}^{n \times n}\right) \backslash L^{*}\left([a, b], \mathbb{R}^{n \times n}\right) \subseteq L\left([a, b], \mathbb{R}^{n \times n}\right) \backslash R\left([a, b], \mathbb{R}^{n \times n}\right)$, the preceding theorem implies that $L\left([a, b], \mathbb{R}^{n \times n}\right) \backslash R\left([a, b], \mathbb{R}^{n \times n}\right)$ is $\mathfrak{c}$-algebrable.

\section{Product integrability of Denjoy integrable matrix-valued functions}

The following definition generalizes the concept of Denjoy product integration.

Definition 4.1. Consider the function $A:[a, b] \rightarrow \mathbb{R}^{n \times n}$ and let $[c, d] \subset[a, b]$. The oscilation of $A$ on the interval $[c, d]$ is the number

$$
\operatorname{osc}(A,[c, d])=\sup \left\{\left\|A\left(\xi_{1}\right)-A\left(\xi_{2}\right)\right\|: \xi_{1}, \xi_{2} \in[c, d]\right\}
$$

The abbreviations $A C, B V$ and $A C G$ stand for "absolutely continuous",

"bounded variations" and "generalized absolutely continiuous", respectively.

Definition 4.2. Let $A:[a, b] \rightarrow \mathbb{R}^{n \times n}$ and $E \in[a, b]$.

1. The strong variation of $F$ on $E$ is defined by

$$
V_{*}(F, E)=\sup \left\{\sum_{i=1}^{n} \operatorname{osc}\left(F,\left[c_{i}, d_{i}\right]\right)\right\},
$$

where the supremum is taken over all finite collections $\left\{\left[c_{i}, d_{i}\right]: 1 \leq i \leq n\right\}$ of non-overlapping intervals that have endpoints in $E$. 
2. The function $F$ is of bounded variation in the restricted sense on $E$ (briefely $A$ is $B V_{*}$ on $E$ ) if $V_{*}(F, E)$ is finite.

3. The function $A$ is absolutely continuous in the restricted sense on $E$ (briefely $A$ is $A C_{*}$ on $E$ ) if for each $\varepsilon>0$, there exists $\delta>0$ such that $\sum_{i=1}^{n} \operatorname{osc}\left(A,\left[c_{i}, d_{i}\right]\right)<\varepsilon$, whenevere $\left\{\left[c_{i}, d_{i}\right]: 1 \leq i \leq n\right\}$ is a finite collection of non-overlapping intervals that have endpoints in $E$ and satisfy $\sum_{i=1}^{n}\left(d_{i}-c_{i}\right)<\delta$.

4. The function $A$ is generalized absolutely continuous in the restricted sense on $E$ (briefely $A$ is $A C G_{*}$ on $E$ ) if $\left.A\right|_{E}$ is continuous on $E$ and $E$ can be written as a countable union sets on each of which $A$ is $A C_{*}$.

Note that in general, $V(F, E) \leq V_{*}(F, E)$ and hence $A$ is $B V(A C, B V G, A C G)$ on $E$ if it is $B V_{*}\left(A C_{*}, B V G_{*}, A C G_{*}\right)$ on $E$.

Definition 4.3. The function $A:[a, b] \rightarrow \mathbb{R}^{n \times n}$ is Denjoy integrable on $[a, b]$ if there exists an ACG $G_{*}$ function $\mathcal{A}:[a, b] \rightarrow \mathbb{R}^{n \times n}$ such that $\mathcal{A}^{\prime}=A$ a.e. on $[a, b]$.

Theorem 4.4. [15, Theorem 6.2] Let $F:[a, b] \rightarrow \mathbb{R}^{n \times n}$ and $E \subseteq[a, b]$.

(1) If $F$ is $A C\left(A C G, A C_{*}, A C G_{*}\right)$ on $E$, then $F$ is $B V\left(B V G, B V_{*}, B V G_{*}\right)$ on $E$.

(2) If $F$ is $B V_{*}$ on $E$, then $F$ is $B V_{*}$ on $\bar{E}$.

(3) Suppose that $E$ is closed with $a, b \in E$ and let $G$ be the linear extension of $F$ to $[a, b]$. If $F$ is $B V(A C)$ on $E$, then $G$ is $B V(A C)$ on $[a, b]$.

Remark 4.1. Let $P$ be a perfect set. A perfect portion of $P$ is a set of the form $P \cap[c, d]$ where $P \cap(c, d) \neq \varnothing$, $c, d \in P$, and $P \cap[c, d]$ is a perfect set.

Theorem 4.5. [15, Theorem 6.10] Suppose that $F:[a, b] \rightarrow \mathbb{R}^{n \times n}$ is $A C G\left(A C G_{*}\right)$ on $[a, b]$ and let $E \subset[a, b]$ be a perfect set. Then there is a perfect portion $E \cap[c, d]$ of $E$ such that $F$ is $A C\left(A C_{*}\right)$ on $E \cap[c, d]$.

( Note that in this case, each subinterval of $[a, b]$ contains an interval on which the function $F$ is $A C\left(A C_{*}\right)$. The endpoints of all the intervals on which $F$ is $A C\left(A C_{*}\right)$ form a dence set in $\left.[a, b]\right)$.

We recall that the next Lemma and proposition are mentioned in [15] as exercises.

Lemma 4.1. Let $F:[a, b] \rightarrow \mathbb{R}^{n \times n}$, and $E$ be a closed set with bounds $a$ and $b$, and let $[a, b]-E=$ $\bigcup_{n=1}^{\infty}\left(a_{n}, b_{n}\right)$. Suppose that $G$ is the linear extension of $F$ from $E$ to $[a, b]$ and $c \in E$. Then $\frac{G(x)-G(c)}{x-c}$ is between $\frac{F\left(a_{n}\right)-F(c)}{a_{n}-c}$ and $\frac{F\left(b_{n}\right)-F(c)}{b_{n}-c}$ for each $x \in\left(a_{n}, b_{n}\right)$. In particular, if $c$ is two-sided limit point of $E$ and $F$ is differentiable at $c$, then $G$ is differentiable at $c$ and $G^{\prime}(c)=F^{\prime}(c)$. 
Proof. First we note that $G=F$ on $E$ and $G$ is linear on each of the intervals contiguous to $E$. For each $x \in\left[a_{n}, b_{n}\right]$, we have

$$
G(x)=\frac{F\left(b_{n}\right)-F\left(a_{n}\right)}{b_{n}-a_{n}}\left(x-a_{n}\right)+F\left(a_{n}\right)
$$

and hence an easy calculation completes the proof.

Proposition 4.1. Suppose that $A:[a, b] \rightarrow \mathbb{R}^{n \times n}$ is Denjoy integrable on $[a, b]$. Then $[a, b]=\cup_{n=1}^{\infty} E_{n}$ where each $E_{n}$ is closed and $A$ is Lebesgue integrable on each $E_{n}$.

Proof. By the hypothesis, there exists an $A C G_{*}$ function $\mathcal{A}:[a, b] \rightarrow \mathbb{R}^{n \times n}$ such that $\mathcal{A}^{\prime}=A$ a.e. on $[a, b]$, and we can write $[a, b]=\cup_{n=1}^{\infty} E_{n}$, where $\mathcal{A}$ is $A C_{*}$ on each $E_{n}$. By Theorem 4.4 we can assume that each $E_{n}$ is closed. Then by Theorem 4.5 there exists a perfect portion $E_{n} \cap[c, d]$ of $E_{n}$ for $n \in \mathbb{N}$, such that $\mathcal{A}$ is $A C_{*}$ on $E_{n} \cap[c, d]$. Let $G:[c, d] \rightarrow \mathbb{R}^{n \times n}$ be the linear extension of $\left.\mathcal{A}\right|_{E_{n} \cap[c, d]}$ to $[c, d]$. By part 3 of Theorem 4.4, $G$ is $A C$ on $[c, d]$. So the function $G^{\prime}$ exists a.e. and is Lebesgue integrable on $[c, d]$. But by Lemma 4.1 $\mathcal{A}^{\prime}=G^{\prime}=A$ a.e. on $E_{n} \cap[c, d]$, so the function $A$ is Lebesgue integrable.

Theorem 4.6. Let $A:[a, b] \rightarrow \mathbb{R}^{n \times n}$ be Denjoy integrable on $[a, b]$, then it is product integrable.

Proof. Let $D\left([a, b], \mathbb{R}^{n \times n}\right)$ be endowed by the norm $\|A\|=(D) \int_{a}^{b}\|A(t)\| d t$, where (D) stands for the Denjoy integral. By Proposition 4.1 there exists subsets $E_{n}$ such that $[a, b]=\cup_{n=1}^{\infty} E_{n}$ where for each $n \in \mathbb{N}, E_{n}$ is non-overlapping, closed and $A$ is Lebesgue integrable on $E_{n}$. Let $A_{n}$ be the restriction of $A$ to $E_{n}$ for each $n \in \mathbb{N}$. Then each $A_{n}$ is Lebesgue integrable and so product integrable and hence for each $A_{n}$ there exists a sequense of step functions $\left\{A_{n_{k}}\right\}_{k=1}^{\infty}$ such that $A_{n_{k}}: E_{n} \rightarrow \mathbb{R}^{n \times n}$ and

$$
\lim _{k \rightarrow \infty}\left\|A_{n_{k}}-A_{n}\right\|_{E_{n}}=\lim _{k \rightarrow \infty} \int_{E_{n}}\left\|A_{n_{k}}(x)-A_{n}(x)\right\| d x=0
$$

For each $n$, put $a_{n}=\inf E_{n}$ and $b_{n}=\sup E_{n}$, so both $a_{n}, b_{n}$ are in $E_{n}$. Thus for each $E_{n}$ there exist $t_{0}, t_{1}, \ldots, t_{n}$ such that

$$
t_{0}=a_{n} \leq t_{1} \leq \ldots \leq t_{n}=b_{n}
$$

and $A_{n_{k}}$ is constant on $\left(t_{k-1}, t_{k}\right)$ for $k=1, \ldots, n$. Now let $\left\{B_{k}\right\}_{k=1}^{\infty}$ be a sequence of step functions on $[a, b]$ such that $[a, b]=\bigcup_{n=1}^{\infty} E_{n}$ and $B_{k}=A_{n_{k}}$ on each $E_{n}$. Then by Dominated Convergence Theorem we have the followings:

$$
\begin{aligned}
\lim _{k \rightarrow \infty}\left\|B_{k}-A\right\|_{1} & =\lim _{k \rightarrow \infty} \int_{a}^{b}\left\|B_{k}(x)-A(x)\right\| d x \\
& =\lim _{k \rightarrow \infty} \sum_{n=1}^{\infty} \int_{E_{n}}\left\|A_{n_{k}}(x)-A_{n}(x)\right\| d x=0
\end{aligned}
$$


i.e., $B_{k}$ converges to $A$ also in the norm of space $D\left([a, b], \mathbb{R}^{n \times n}\right)$ and hence by $[16$, Theorem 3.5 .5$] \underset{k \rightarrow \infty}{\lim }(I+$ $\left.B_{k}(x)\right) d x \prod_{a}^{b}$ exists. So the proof is complete.

\section{5. $\mathfrak{c}$-Algebrability of the Set of Denjoy PRoduct integrable}

In this section, some pathological properties (more precisely algebrability) of sets of product integrable functions contained in $D\left([a, b], \mathbb{R}^{n \times n}\right) \backslash L\left([a, b], \mathbb{R}^{n \times n}\right)$ are investigated. First we note that a matrix $A=$ $\left\{a_{i j}\right\}_{i, j=1}^{n}$ is called regular if it has a nonzero determinant.

Definition 5.1. A function $A:[a, b] \rightarrow \mathbb{R}^{n \times n}$ is called Perron product integrable if there is a regular matrix $B \in \mathbb{R}^{n \times n}$ such that for every $\varepsilon>0$ there is a function $\delta:[a, b] \rightarrow(0, \infty)$ such that $\|B-P(A, D)\|<\varepsilon$ for every $\delta$-fine partition $D$ of $[a, b]$.

Theorem 5.2. Consider the function $A:[a, b] \rightarrow \mathbb{R}^{n \times n}$ in $D\left([a, b], \mathbb{R}^{n \times n}\right)$. Then

$$
\prod_{a}^{b} e^{A(t) d t}=(I+A(t) d t) \prod_{a}^{b}
$$

Proof. By [10, Theorem 2.12] and [15, Theorem 11.2], the proof is clear.

Corollary 5.1. If $A:[a, b] \rightarrow \mathbb{R}^{n \times n}$ is product integrable function, then exp $\circ(A)$ is product integrable function.

Theorem 5.3. The set of product integrable functions is strongly c-algebrable.

Proof. By Corollary 5.1 and Theorem 3.2 the proof follows.

Proposition 5.1. [15, Theorem 7.11] Suppose that $f:[a, b] \rightarrow \mathbb{R}$ is Denjoy integrable on each subinterval $[c, d] \subseteq(a, b)$. If $\int_{c}^{d} f$ converges to a finite limit as $c \rightarrow a^{+}$and $d \rightarrow b^{-}$, then $f$ is Denjoy integrable on $[a, b]$ and $\int_{a}^{b} f=\lim _{\substack{c \rightarrow a^{+} \\ d \rightarrow b^{-}}} \int_{c}^{d} f$.

Theorem 5.4. The set of $D\left([a, b], \mathbb{R}^{n \times n}\right) \backslash L\left([a, b], \mathbb{R}^{n \times n}\right)$ is strongly $\mathfrak{c}$-algebrable.

Proof. Let $\sum_{n=1}^{\infty} c_{n}$ be a nonabsolutely convergent series of real numbers and let $I_{n}=\left(2^{-n}, 2^{-n+1}\right), n \in \mathbb{N}$. Define the function $A:[0,1] \rightarrow \mathbb{R}^{n \times n}$ by $A(x)=\left(a_{i j}(x)\right)_{i, j=1}^{n}$, such that for each $i, j=1,2, \ldots, n$

$$
\left(a_{i j}(x)\right)= \begin{cases}2^{n} c_{n} & x \in I_{n} \\ 0 & \text { otherwise. }\end{cases}
$$

Note that

$$
\int_{0}^{1}\left|a_{i j}(x)\right| d x=\sum_{n=1}^{\infty} \int_{I_{n}}\left|a_{i j}(x)\right| d x=\sum_{n=1}^{\infty}\left|2^{-n} c_{n} 2^{n}\right|=\sum_{n=1}^{\infty}\left|c_{n}\right|=\infty .
$$


Hence neither $a_{i j}$ nor $A$ is Lebesgue integrable on $[0,1]$. Now we are going to show that $A$ is Denjoy integrable on $[0,1]$. For each $0<\alpha<1$ both of functions $a_{i j}$ and $A$ are bounded on $[\alpha, 1]$, so they are Lebesgue integrable on $[\alpha, 1]$. Let $B(x)=\int_{x}^{1} a_{i j}$ for each $x \in(0,1]$. The function $B$ is linear on each $I_{n}$. It follows that $B(x)$ is between $B\left(2^{-n}\right)$ and $B\left(2^{n}\right)$ for each $x \in I_{n}$. Now $B\left(2^{-n}\right)=\sum_{k=1}^{n} c_{k}$ and $\lim _{n \rightarrow \infty} B\left(2^{-n}\right)=\sum_{k=1}^{\infty} c_{k}$.

Therefore $\lim _{x \rightarrow 0^{+}} B(x)=\sum_{n=1}^{\infty} c_{n}$ and according to Proposition 5.1, $a_{i j}$ is Denjoy integrable on $[0,1]$ for each $i, j=1,2, \ldots, n$. Thus for each $a_{i j}(x)$ there exists an $A C G_{*}$ function $f_{i j}$ such that $f^{\prime}{ }_{i j}(x)=a_{i j}(x)$ a.e. on $x \in[0,1]$. Now put $F(x)=\left(f_{i j}(x)\right)_{i, j=1}^{n}$ for each $x \in[0,1]$. So

$$
F^{\prime}(x)=\left(f^{\prime}{ }_{i j}(x)\right)_{i, j=1}^{n}=\left(a_{i j}(x)\right)_{i, j=1}^{n}=A(x) \text { a.e. on }[0,1] .
$$

Hence $A$ is Denjoy integrable on $x \in[0,1]$. One can see easily that $\exp \circ a_{i j}$ is Denjoy integrable and so is $\exp \circ A$. Thus by Theorem 3.2 the proof is complete.

\section{REFERENCES}

[1] R. Aron, V. I. Gurariy and J. B. Seoane-Sepúlveda, lineability and spaceability of sets of functions on $\mathbb{R}$, Proc. Amer. Math. Soc. 133 (3) (2005), 795-803.

[2] R. Aron and J. B. Seoane-Sepúlveda, Algebrability of the set of everywhere surjective functions on $\mathbb{C}$, Bull. Belg. Math. Soc. Simon Stevin 14 (1) (2007), 25-31.

[3] M. Balcerzak, A. Bartoszewicz and M. Filipczac, Nonseparable spaceability and strong algebrability of sets of continuous singular functions, J. Math. Anal. Appl. 407 (2) (2013), 263-269.

[4] A. Bartoszewicz, M. Bieniea, M. Filipczac and S. Glab, Strong c-algebrability of strong Sierpinski-Zygmund, smooth nowhere analytic and other sets of functions, J. Math. Anal. Appl. 412 (2) (2015), 620-630.

[5] A. Bartoszewicz and S. Glab, Strong c-algebrability of sets of sequences and functions, Proc. Amer. Math. Soc. 141 (2013), 827-835.

[6] A. Farokhinia, Algebrability of space of quasi-everywhere surjective functions. B. Math. Anal. Appl. 6 (6) (2014), 38-43.

[7] A. Farokhinia, Lineability of Denjoy integrable function, J. Math. Ext. 11 (1) (2017), 57-65.

[8] A. Farokhinia, Lineability of space of quasi-everywhere surjective functions. J. Math. Ext. 6 (3) (2013), 45-51.

[9] V. I. Gurariy and L. Qurta, On lineability of sets of continuous functions, J. Math. Anal. Appl. (1) (2004), 62-72.

[10] J. Jarník and J. Kurzweil, A general form of the product integral and linear ordinary, Czech. Math. J. 37 (4) (1987), 642-659.

[11] F. R. Riesz, Sur lintegrale de Lebesgue. Acta Mathematica 42 (1919), 191-205.

[12] L. Schlesinger, Neue Grandlagen fur einen Infinittesimalkul der Matrizen, Mathematische Zeitschrift 33 (1931), 33-61.

[13] A. Slavík and S̆. Schwabic, Henstock-Kurzweil and McShane product integrals; descriptive definations, Czech. Math. J. 58 (133) (2008), 241-269.

[14] S̆. Schwabic, Bochner product integration, Math. Bohem. 119 (1994), 305-335.

[15] R. Gordon, The integrals of Lebesgue, Denjoy, Perron and Henstock, American Mathematical Society, 1994.

[16] A. Slavík, Product integration, its history and applications, Matfyzpress, Prague, 2007.

[17] V. Volterra and B. Hostinsky, Operations infinitesimales lineaires, Gauthier-Villars, Paris, 1938. Adison-Wesley. Publishing Company 1979. 